Homology, Homotopy and Applications, vol.4(2), 2002, pp.117-139

\title{
AN ALGEBRAIC MODEL FOR HOMOTOPY FIBERS
}

\author{
NICOLAS DUPONT AND KATHRYN HESS
}

(communicated by Larry Lambe)

Abstract

Let $F$ be the homotopy fiber of a continuous map $f$ : $X @>>>$, and let $R$ be a commutative, unitary ring. Given a morphism of chain Hopf algebras that models $(\Omega f)_{\sharp}$ : $C_{*}(\Omega X ; R) @>>>C_{*}(\Omega Y ; R)$, we construct a cochain algebra that models $C^{*}(F ; R)$. We explain how to simplify the model for certain large classes of maps $f$ and provide examples of the application of our model.

\section{To Jan-Erik Roos on his sixty-fifth birthday}

\section{Introduction}

Homotopy fibers of continuous maps play an essential role in algebraic topology. In this article we provide a tool for calculating the cohomology algebra of the homotopy fiber $F$ of a continuous map $f$ in terms of a morphism of chain Hopf algebras that models $(\Omega f)_{\sharp}: C U_{*}(\Omega X ; R) @>>>C U_{*}(\Omega Y ; R)$, where $R$ is a commutative, unitary ring and $C U_{*}$ denotes cubical chains. More precisely our input data consist of a commuting diagram

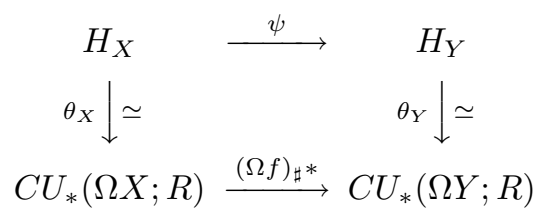

in which $\psi$ is a morphism of cocommutative, coassociative chain Hopf algebras of finite type, and $\theta_{X}$ and $\theta_{Y}$ induce isomorphisms of Hopf algebras in cohomology. Let $C_{X}$ and $C_{Y}$ denote the $R$-duals of $H_{X}$ and $H_{Y}$. Given (0.1), we construct a cochain algebra $\Omega C_{X} \odot C_{Y}$, a "twisted extension" of the cobar construction on $C_{X}$, denoted $\Omega C_{X}$, by $C_{Y}$, that admits a natural filtration by wordlength in the first factor. We prove the existence of a morphism of cochain complexes $\zeta: \Omega C_{X} \odot C_{Y} @>>>$ $C^{*}(F ; R)$ that induces an isomorphism of algebras

$$
E_{0}\left(H^{*} \zeta\right): E_{0}\left(H^{*}\left(\boldsymbol{\Omega} C_{X} \odot C_{Y}\right)\right) @>\cong>E_{0}\left(H^{*}(F ; R)\right),
$$

Received March 6, 2001, revised August 20, 2001; published on July 12, 2002.

2000 Mathematics Subject Classification: 55P99, 55U15, 55T20.

Key words and phrases: homotopy fiber, algebraic model, Adams-Hilton model.

(c) 2002, Nicolas Dupont and Kathryn Hess. Permission to copy for private use granted. 
where $E_{0}$ refers to the wordlength-filtration on the one hand and to the usual Eilenberg-Moore filtration on the other. We also establish conditions under which $H^{*} \zeta$ itself is an algebra morphism.

The methods we apply are closely related to those we developed in $[\mathbf{5}],[\mathbf{6}],[\mathbf{7}]$, and [8], where we established a general theory of noncommutative algebraic models of topological spaces and continuous maps, which we then applied to building a model of the free loop space. Whereas our earlier work assumed field coefficients at all times, the constructions we describe in this article can be carried out over any commutative, unitary ring $R$.

In the first section of this article we explain how a chain Hopf algebra model like (0.1), for an injective map $f$, gives rise to a commuting diagram in the category of cochain algebras

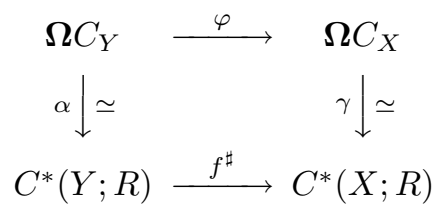

in which $\alpha$ and $\gamma$ both induce isomorphisms in cohomology. Our starting point in the second section is a chain Hopf algebra $H$ together with a chain algebra morphism $H @>>>C U_{*}(\Omega Y ; R)$ inducing an isomorphism of Hopf algebras in homology. We then construct in a cochain algebra $\Omega C \odot C$, a "twisted extension" of $\boldsymbol{\Omega} C$ by $C$, together with a quasi-isomorphism $\Omega C \odot C @>>C^{*}\left(P_{*} Y ; R\right)$, where $C$ is the $R$-dual of $H$. We form the "push-out" of these two constructions in section three, obtaining the desired model $\zeta: \Omega C_{X} \odot C_{Y} @>>>C^{*}(F ; R)$. In the last section of the article, we present two elementary applications of our model, as a foretaste of the results we will publish in a future article.

\section{Notation and terminology}

- A morphism in a category of differential graded objects that induces an isomorphism in homology is denoted @ $>\simeq>>$.

- If $X$ is a topological space and $R$ is a commutative, unitary ring, then $C U_{*}(X ; R)$ is the complex of cubical chains on $X$ with coefficients in $R$, while $C_{*}(X ; R)$ and $C^{*}(X ; R)$ are the singular chain and cochain complexes with coefficients in $R$.

- If $X$ is a pointed topological space, then $\Omega X$ denotes the space of Moore loops on $X$, which is a strictly associative $\mathrm{H}$-space.

- If $R$ is a commutative ring and $M=\bigoplus_{i \in \mathbb{N}} M_{i}$ is a graded $R$-module, then $M^{\star}$ denotes its $R$-dual, i.e., $M^{\star}=\bigoplus_{i \in \mathbb{N}}\left(M^{\star}\right)^{i}$ where $\left(M^{\star}\right)^{i}=H_{o m}\left(M_{i}, R\right)$. Furthermore the $R$-dual of a homomorphism $h$ of $R$-modules is denoted $h^{\star}$.

- If $M=\bigoplus_{i} M_{i}$ is a graded $R$-module, then $s M$ denotes the graded module with $(s M)_{i} \cong M_{i-1}$, while $s^{-1} M$ denotes the graded module with $\left(s^{-1} M\right)_{i} \cong M_{i+1}$. If, on the other hand, we consider a module with upper grading, $M=\bigoplus_{i} M^{i}$, then $s M$ denotes the graded module with $(s M)^{i} \cong M^{i+1}$, while $s^{-1} M$ denotes the graded module with $\left(s^{-1} M\right)^{i} \cong M^{i-1}$. In both cases, given a homogeneous 
element $x$ in $M$, we write $s x$ and $s^{-1} x$ for the corresponding elements of $s M$ and $s^{-1} M$.

- If $x$ is a homogeneous element of a graded module $M$, we denote its degree by $|x|$, unless it is used as an exponent, in which case we drop the bars.

- Given a connected (co)chain algebra $A$, we denote the bar construction on $A$ by $\mathcal{B} A$. The cobar construction on a 1-connected (co)chain algebra $C$ is denoted $\Omega C$. Recall that the underlying coalgebra of $\mathcal{B} A$ is the cofree coalgebra $\perp s \bar{A}$, while the underlying algebra of $\Omega C$ is the free algebra $T s^{-1} \bar{C}$, where $\bar{A}$ denotes the augmentation ideal of $A$ and $\bar{C}$ the coaugmentation coideal of $C$.

- The underlying graded $R$-module of any (co)chain (co)algebra in this article is assumed to be free.

\section{An $R$-model of a continuous injection}

Let $f: X @>>>Y$ be a continuous map, and let $R$ be a commutative, unitary ring. We present in this section a tool for computing the homomorphism of graded algebras induced by $f$ on cohomology with coefficients in $R$, at least when certain conditions on $f$ are satisfied.

Recall from [2] that a Hopf algebra up to homotopy is an associative chain algebra $H$ endowed with a morphism of chain algebras $\Delta: H @>>>B H$ that is coassociative and cocommutative up to homotopy of chain algebras and such that the augmentation of $H$ is a counit up to homotopy of chain algebras with respect to $\Delta$. If $Z$ is any topological monoid, e.g., if $Z=\Omega X$ for some pointed space $X$, then $C U_{*}(Z ; R)$ is a Hopf algebra up to homotopy.

Definition. A strict Hopf model for $f$ over $R$ consists of a diagram

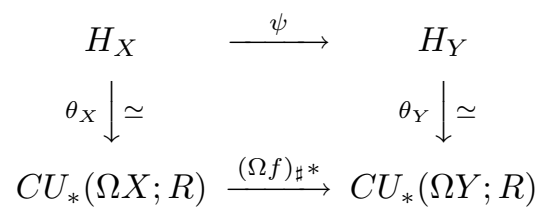

such that

1. $H_{X}$ and $H_{Y}$ are chain Hopf algebras that are cocommutative, coassociative and of finite type;

2. $\psi$ is a morphism of chain Hopf algebras;

3. $\theta_{X}$ and $\theta_{Y}$ are quasi-isomorphisms of Hopf algebras up to homotopy; and

4. $\theta_{Y} \psi$ and $(\Omega f)_{\sharp} \theta_{X}$ are homotopic as maps of chain algebras.

The strict Hopf model (1.1) is injective if $\psi$ is an injection.

Existence. Suppose that $R$ is a subring of $\mathbb{Q}$, and let $p$ be the smallest prime that is not invertible in $R$. Anick proved in $[2,8.4]$ that if $X$ and $Y$ are CW-complexes with trivial $r$-skeleton and of dimension at most $r p$, then any continuous map $f$ : $X @>>>Y$ possesses a strict Hopf model in which $H_{X}$ and $H_{Y}$ are enveloping 
algebras of free differential graded Lie algebras. The spaces $X$ and $Y$ are then examples of the $p$-Anick spaces we have studied elsewhere $([\mathbf{7}],[\mathbf{8}])$.

If $f$ is the inclusion of a subcomplex into a larger CW-complex, then there is a strict Hopf model (1.1) of $f$ in which $\psi$ is the inclusion of a subHopf algebra.

Given an injective, continuous map $f$ that possesses a strict Hopf model, our goal in this section is to construct a strict cochain algebra model of $f$ over $R$, i.e., a commuting diagram in the category of cochain algebras over $R$

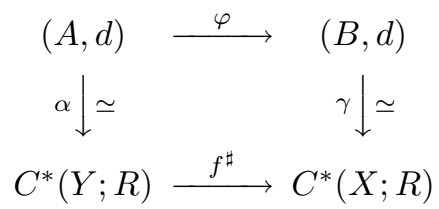

where $C^{*}(-; R)$ denotes the singular cochains with coefficients in $R$.

Let $C_{X}$ and $C_{Y}$ denote the $R$-duals of $H_{X}$ and $H_{Y}$. Since $H_{X}$ and $H_{Y}$ are of finite type, their duals are cochain Hopf algebras. Furthermore, $\left(\mathcal{B} H_{X}\right)^{\star} \cong \Omega C_{X}$ and $\left(\mathcal{B} H_{Y}\right)^{\star} \cong \Omega C_{Y}$ as cochain algebras, also for reasons of finite type.

By Lemma 4.3(ii) in [10], $\mathcal{B} \theta_{X}$ and $\mathcal{B} \theta_{Y}$ are also quasi-isomorphisms, since all objects in the diagram are $R$-semifree. Proposition 2.3(ii) in the same article then implies that their $R$-duals, $\left(\mathcal{B} \theta_{X}\right)^{\star}$ and $\left(\mathcal{B} \theta_{Y}\right)^{\star}$, are quasi-isomorphisms as well.

Thus, if we apply the bar construction to diagram 2.1 and then dualize, we obtain the diagram

$$
\begin{array}{ccc}
\Omega C_{X} & \longleftarrow \varphi & \Omega C_{Y} \\
\left(\mathcal{B} \theta_{X}\right)^{\star} \uparrow \simeq & \left(\mathcal{B} \theta_{Y}\right)^{\star} \uparrow \simeq \\
\left(\mathcal{B} C U_{*}(\Omega X ; R)\right)^{\star} \stackrel{\left(\mathcal{B}(\Omega f)_{\sharp}\right)^{\star}}{\longleftarrow} & \left(\mathcal{B} C U_{*}(\Omega Y ; R)\right)^{\star}
\end{array}
$$

where $\varphi=\boldsymbol{\Omega}\left(\psi^{\star}\right)$. Since both the bar construction and dualisation preserve quasiisomorphisms, which are the weak equivalences in each of the model categories involved, they induce functors on the homotopy categories. Consequently, $\varphi\left(\mathcal{B} \theta_{Y}\right)^{\star}$ and $\left(\mathcal{B} \theta_{X}\right)^{\star}\left(\mathcal{B}(\Omega f)_{\sharp}\right)^{\star}$ are homotopic as cochain algebra maps.

Applying Proposition $2.9(\mathrm{c})$ in $[\mathbf{2}]$ to the cochain algebra morphisms $\left(\mathcal{B} \theta_{X}\right)^{\star}$ and $\left(\mathcal{B} \theta_{Y}\right)^{\star}$, we obtain homotopy right inverses $\gamma^{\prime}: \Omega C_{X} @>\simeq>>\left(\mathcal{B} C_{*}(\Omega X ; R)\right)^{\star}$ and $\alpha^{\prime}: \Omega C_{Y} @>\simeq>>\left(\mathcal{B} C_{*}(\Omega Y ; R)\right)^{\star}$, i.e., $\left(\mathcal{B} \theta_{X}\right)^{\star} \gamma^{\prime} \sim I d_{\Omega C_{X}}$ and $\left(\mathcal{B} \theta_{Y}\right)^{\star} \alpha^{\prime} \sim$ $I d_{\Omega C_{Y}}$. Thus

$$
\left(\mathcal{B} \theta_{X}\right)^{\star} \gamma^{\prime} \varphi \sim \varphi\left(\mathcal{B} \theta_{Y}\right)^{\star} \alpha^{\prime} \sim\left(\mathcal{B} \theta_{X}\right)^{\star}\left(\mathcal{B}(\Omega f)_{\sharp}\right)^{\star} \alpha^{\prime} .
$$

Since $\Omega C_{Y}$ is free and $\left(\mathcal{B} \theta_{X}\right)^{\star}$ is a quasi-isomorphism, Lemma 2.10 in [An] implies that $\gamma^{\prime} \varphi \sim\left(\mathcal{B}(\Omega f)_{\sharp}\right)^{\star} \alpha^{\prime}$.

In [1], Adams proved the existence for all simply-connected, based spaces $X$ and for all $R$ of a quasi-isomorphism of chain algebras $\Omega C_{*}(X ; R) @>>C U_{*}(\Omega X ; R)$. He showed furthermore that the isomorphism induced in homology was natural in $X$. Félix, Halperin and Thomas improved upon Adams's result in [9], establishing that the Adams equivalence can be constructed so that it is natural already on the chain level. Applying the bar construction to Adams's equivalence and using the natural quasi-isomorphism of chain coalgebras $C_{*}(X ; R) @>\simeq>>\mathcal{B} \Omega C_{*}(X ; R)$ given by 
the adjunction, we obtain a natural quasi-isomorphism $A_{X}: * C_{*}(X ; R) @>\simeq>>$ $\mathcal{B} C U_{*}(\Omega X ; R)$.

Combining the $R$-dual of Adams's equivalence with the diagram derived from (1.2) using $\alpha^{\prime}$ and $\gamma^{\prime}$, we obtain the diagram

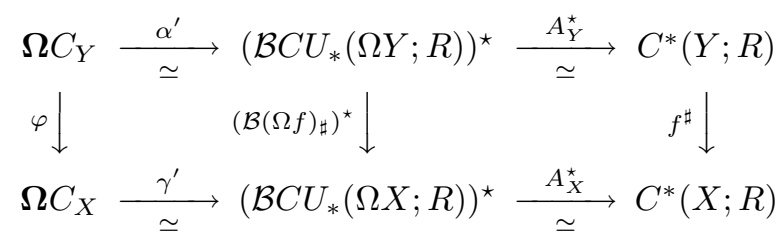

in which the left-hand square commutes up to homotopy of cochain algebras and the right-hand square commutes exactly. Let $\gamma=A_{X}^{\star} \gamma^{\prime}$. A standard homotopy-lifting argument then shows that if $f^{\sharp}$ is surjective, i.e., if $f$ is injective, then there is a cochain algebra map $\alpha: \Omega C_{Y} @>>>C^{*} Y$ such that $f^{\sharp} \alpha=\gamma \varphi$. We have thus constructed a strict cochain algebra model for $f$ over $R$.

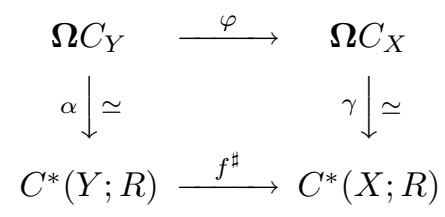

\section{Cochain algebra models of path fibrations}

Let $Y$ be a space for which there exists a cocommutative, coassociative differential graded Hopf algebra of finite type $H$ together with a quasi-isomorphism of Hopf algebras up to homotopy $H @>\simeq>>C U_{*}(\Omega Y ; R)$. Let $C$ denote the $R$-dual of $H$. In [7] we constructed, for $R$ any field, a cochain algebra $\boldsymbol{\Omega}(C \otimes C) \odot C$ together with a quasi-isomorphism $\boldsymbol{\Omega}(C \otimes C) \odot C @>\simeq>>C^{*}\left(Y^{I} ; R\right)$ of $\boldsymbol{\Omega}(C \otimes C)$-bimodules inducing a morphism of graded algebras in cohomology.

In this section we first verify that this construction actually works over any commutative, unitary ring. We then derive an $R$-model for the based path space $P_{*} Y$ from the model for $Y^{I}$.

We begin by defining a $\boldsymbol{\Omega}(C \otimes C)$-bimodule, which is a perturbation of the usual tensor product of cochain complexes $\Omega(C \otimes C) \otimes C$. Let $\partial$ denote the differential on $C$, and let $\bar{\Delta}$ denote its reduced coproduct.

Definition of $(\boldsymbol{\Omega}(C \otimes C) \widetilde{\otimes} C)$. Let $\boldsymbol{\Omega}(C \otimes C) \widetilde{\otimes} C$ denote the graded $R$-module $\boldsymbol{\Omega}(C \otimes C) \otimes C$ endowed with a two-sided, associative action of $\boldsymbol{\Omega}(C \otimes C)$ on $\boldsymbol{\Omega}(C \otimes$ $C) \otimes C$ and a degree +1 endomorphism

$$
D: \boldsymbol{\Omega}(C \otimes C) \otimes C @>>>\boldsymbol{\Omega}(C \otimes C) \otimes C
$$

determined by the conditions that for all $a, b, c$ in $C$ with $\bar{\Delta}(c)=\sum_{j \in J} c_{j} \otimes c^{j}$ :

1. the right action of $\boldsymbol{\Omega}(C \otimes C)$ is free, i.e.,

$$
(1 \otimes c) \cdot\left(s^{-1}(a \otimes b) \otimes 1\right)=(-1)^{c(a+b+1)} s^{-1}(a \otimes b) \otimes c ;
$$


2. the left action of $\Omega(C \otimes C)$ commutes with the right action and is given recursively by

$$
\begin{aligned}
\left(s^{-1}(a \otimes b) \otimes 1\right) \cdot(1 \otimes c)= & s^{-1}(a \otimes b) \otimes c \\
& +(-1)^{b c}\left(s^{-1}(a \otimes c b) \otimes 1-s^{-1}(a c \otimes b) \otimes 1\right) \\
& +\sum_{j \in J}\left[(-1)^{b c_{j}+1}\left(s^{-1}\left(a c_{j} \otimes b\right) \otimes 1\right) \cdot\left(1 \otimes c^{j}\right)\right. \\
& \left.\quad+(-1)^{b c^{j}+c_{j} c^{j}} s^{-1}\left(a \otimes c^{j} b\right) \otimes c_{j}\right] ;
\end{aligned}
$$

3. $D$ extends the differential on $\boldsymbol{\Omega}(C \otimes C)$;

4. the perturbation of $1 \otimes \partial$ is defined recursively by

$$
\begin{aligned}
D(1 \otimes c)-1 \otimes \partial c= & s^{-1}(c \otimes 1)-s^{-1}(1 \otimes c) \\
+\sum_{j \in J} & {\left[\left(s^{-1}\left(c_{j} \otimes 1\right) \otimes 1\right) \cdot\left(1 \otimes c^{j}\right)\right.} \\
& \left.-(-1)^{c_{j} c^{j}} s^{-1}\left(1 \otimes c^{j}\right) \otimes c_{j}\right] ;
\end{aligned}
$$

5. $D$ is defined to commute with the right action of $\Omega(C \otimes C)$, i.e.,

$$
\begin{aligned}
D\left(s^{-1}(a \otimes b) \otimes c\right)= & D s^{-1}(a \otimes b) \otimes c \\
& +(-1)^{c(a+b+1)}(D(1 \otimes c)) \cdot\left(s^{-1}(a \otimes b) \otimes 1\right) .
\end{aligned}
$$

Theorem 3.1 in $[\mathbf{6}]$ states that $\boldsymbol{\Omega}(C \otimes C) \widetilde{\otimes} C$ is indeed a differential graded bimodule over $\boldsymbol{\Omega}(C \otimes C)$, at least so long as $R$ is a field. It is easy to see, however, that the proof of Theorem 3.1 works over any commutative, unitary ring.

Since $C$ is a commutative, associative Hopf algebra, Theorem 3.3 in [7] implies that $\boldsymbol{\Omega}(C \otimes C) \widetilde{\otimes} C$ underlies a cochain algebra where $(1 \otimes c) \cdot\left(1 \otimes c^{\prime}\right)=1 \otimes c c^{\prime}$. We denote this algebra by $\boldsymbol{\Omega}(C \otimes C) \odot C$. Again, though this theorem is stated with field coefficients, it is immediately clear that the proof holds over any commutative, unitary ring.

Let $\mu: C \otimes C @>>>C$ be the product on $C$. Let $\bar{\mu}: \Omega(C \otimes C) \widetilde{\otimes} C @>>>\Omega C$ denote the morphism of free right $\boldsymbol{\Omega}(C \otimes C)$-modules extending $\boldsymbol{\Omega} \mu$ and determined by $\bar{\mu}(1 \otimes c)=0$ for all $c$ in $C$. Then, according to Theorem 3.3 in $[\mathbf{7}], \bar{\mu}$ is a quasiisomorphism of cochain algebras.

Let $\mathcal{D}: Y @>>>Y \times Y$ denote the diagonal map. Consider the diagram of Hopf algebras up to homotopy

$$
\begin{array}{ccc}
H & \stackrel{\Delta}{\longrightarrow} & H \otimes H \\
\theta \downarrow \simeq & E Z \circ(\theta \otimes \theta) \downarrow & \simeq \\
C U_{*}(\Omega Y ; R) & \stackrel{(\Omega \mathcal{D})_{\sharp}}{\longrightarrow} C U_{*}(\Omega(Y \times Y) ; R)
\end{array}
$$

where $E Z$ denotes the Eilenberg-Zilber equivalence. The coassociativity and cocommutativity of $H$ imply that (2.1) is a strict Hopf model. Since the diagonal map $\mathcal{D}$ 
is injective, we can apply the construction in section 1 to obtain the following strict cochain algebra model.

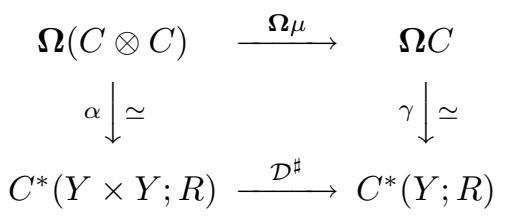

Let $q: Y^{I} @>>>Y \times Y$ denote the path fibration, i.e., $q(\lambda)=(\lambda(0), \lambda(1))$, and let $p: Y @>>>Y^{I}$ denote the map sending a point $y$ of $Y$ to the constant map at $y$. Consider the following commuting diagram in the category of cochain algebras, in which $\iota$ denotes the canonical inclusion.

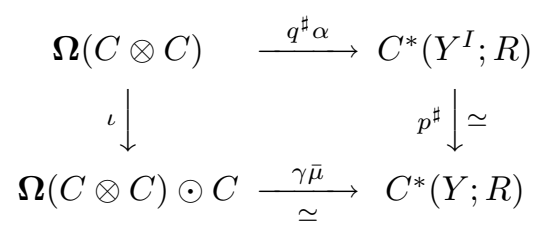

Since $p^{\sharp}$ is a surjective quasi-isomorphism, $\gamma \bar{\mu}$ is a quasi-isomorphism and $\Omega(C \otimes C) \odot$ $C$ is a semi-free right $\boldsymbol{\Omega}(C \otimes C)$-module, $\gamma \bar{\mu}$ lifts through $p^{\sharp}$ to a quasi-isomorphism of right $\boldsymbol{\Omega}(C \otimes C)$-modules $\beta: \boldsymbol{\Omega}(C \otimes C) \odot C @>\simeq>>C^{*} Y^{I}$.

Observe that $\beta$ induces an isomorphism of graded algebras in cohomology, since $H^{*} p$ and $H^{*}(\gamma \bar{\mu})$ are homomorphisms of graded algebras and $H^{*} \beta=\left(H^{*} p\right)^{-1} H^{*}(\gamma \bar{\mu})$. We have therefore constructed a quasi-R-model of $q$, i.e., a commuting diagram of cochain algebras

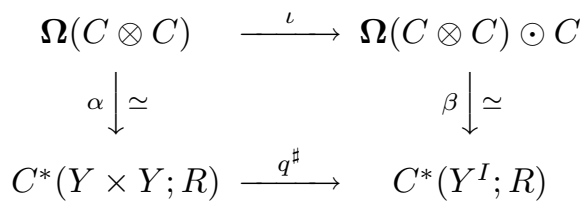

in which $\beta$ is a morphism of right $\boldsymbol{\Omega}(C \otimes C)$-modules such that $H^{*} \beta$ is an algebra homomorphism, and all other morphisms are morphisms of cochain algebras.

Let $y_{0}$ be the basepoint of $Y$, and let $P_{*} Y=\left\{\lambda \in Y^{I} \mid \lambda(0)=y_{0}\right\}$. Our next goal is to construct a model similar to (2.3) for the based path fibration $\bar{q}: P_{*} Y @>>>$ $Y: \lambda \mapsto \lambda(1)$.

Recall first that $P_{*} Y$ is the pull-back of $q$ and $i: Y @>>>Y \times Y: y \mapsto\left(y_{0}, y\right)$, as in the diagram below.

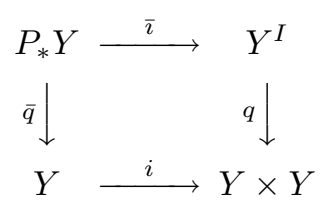

Since $i$ admits an obvious left inverse, we can apply techniques similar to those developed in [5] to the construction of a model for $P_{*} Y$ as a "push-out" of the model (2.3) of $q$ and a model of $i$. 
We build a model of $i$ as follows. Let $\eta: R @>>>H$ denote the unit of $H$. Consider the diagram of Hopf algebras up to homotopy

$$
\begin{array}{ccc}
H & \stackrel{\eta \otimes I d_{H}}{\longrightarrow} & H \otimes H \\
\theta \mid \simeq & E Z \circ(\theta \otimes \theta) \mid \simeq \\
C U_{*}(\Omega Y ; R) \stackrel{(\Omega i)_{\sharp}}{\longrightarrow} C U_{*}(\Omega(Y \times Y) ; R)
\end{array}
$$

where $E Z$ denotes the Eilenberg-Zilber equivalence. The coassociativity and cocommutativity of $H$ imply that (2.4) is a strict Hopf model. Since the inclusion $i: Y @>>>\times Y$ is injective, we can apply the construction in section 1 to obtain the following strict cochain algebra model, where $\rho$ is the $R$-dual of $\eta \otimes I d_{H}$.

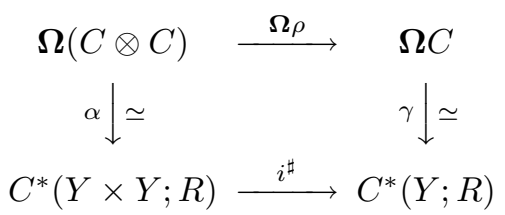

Note that we can ensure that morphisms $\alpha$ and $\gamma$ in (2.5) are the same as those in (2.2) by applying the standard homotopy lifting argument mentioned in section 1 to the diagram

$$
\begin{array}{ccc}
\boldsymbol{\Omega}(C \otimes C) & \stackrel{(\boldsymbol{\Omega} \mu, \boldsymbol{\Omega} \rho)}{\longrightarrow} & \boldsymbol{\Omega} C \times \boldsymbol{\Omega} C \\
A_{Y \times Y}^{\star} \alpha^{\prime} \downarrow \simeq & \gamma \times \gamma \downarrow \simeq \\
C^{*}(Y \times Y ; R) & \stackrel{\left(\mathcal{D}^{\sharp}, i^{\sharp}\right)}{\longrightarrow} & C^{*}(Y ; R) \times C^{*}(Y ; R)
\end{array}
$$

where the morphisms are as in diagram (1.3) and $\times$ denotes the (categorical) product of cochain algebras.

We are now prepared to define our candidate for a model of $P_{*} Y$. Let $d$ denote the differential on $\Omega C, \partial$ the differential on $C$ and $\Delta$ the coproduct on $C$.

Definition of $(\Omega C \odot C)$. Let $\boldsymbol{\Omega} C \odot C$ denote the graded $R$-module $\boldsymbol{\Omega} C \otimes C$ endowed with an associative product

$$
\tilde{\mu}:(\boldsymbol{\Omega} C \otimes C) \otimes(\boldsymbol{\Omega} C \otimes C) @>>>\Omega C \otimes C
$$

and a degree +1 endomorphism extending $d$

$$
\widetilde{D}: \Omega C \otimes C @>>>\Omega C \otimes C
$$

determined by the conditions that for all $a, c, c^{\prime}$ in $C$ with $\bar{\Delta}(c)=\sum_{j \in J} c_{j} \otimes c^{j}$ :

1.

$$
\tilde{\mu}\left((1 \otimes c) \otimes\left(s^{-1} a \otimes 1\right)\right)=(-1)^{c(a+1)} s^{-1} a \otimes c ;
$$

2 .

$$
\begin{aligned}
\tilde{\mu}\left(\left(s^{-1} a \otimes 1\right) \otimes(1 \otimes c)\right)= & s^{-1} a \otimes c+s^{-1}(a c) \otimes 1 \\
& +\sum_{j \in J}(-1)^{c_{j} c^{j}} s^{-1}\left(a c^{j}\right) \otimes c_{j} ;
\end{aligned}
$$


3.

$$
\tilde{\mu}\left((1 \otimes c) \otimes\left(1 \otimes c^{\prime}\right)\right)=1 \otimes c c^{\prime} ;
$$

4. the perturbation of $1 \otimes \partial$ is defined by

$$
\widetilde{D}(1 \otimes c)=1 \otimes \partial c-s^{-1} c-\sum_{j \in J}(-1)^{c_{j} c^{j}} s^{-1} c^{j} \otimes c_{j} ;
$$

5. $\widetilde{D}$ is defined to commute with the right action of $\boldsymbol{\Omega} C$, i.e.,

$$
\widetilde{D}\left(s^{-1} a \otimes c\right)=d s^{-1} a \otimes c+(-1)^{c(a+1)}(\widetilde{D}(1 \otimes c)) \cdot\left(s^{-1} a \otimes 1\right) .
$$

Proposition 2.1. As defined above, $\boldsymbol{\Omega} C \odot C=(\boldsymbol{\Omega} C \otimes C, \widetilde{D}, \tilde{\mu})$ is an associative cochain algebra.

Proof. We need to verify that $\widetilde{D}^{2}=0$ and that $\widetilde{D}$ is a derivation with respect to $\tilde{\mu}$. Notice that $\widetilde{D}$ is defined precisely so that

$$
\widetilde{D}(1 \otimes c)=\left(\boldsymbol{\Omega} \rho \otimes I d_{C}\right) D(1 \otimes c)
$$

for all $c \in C$. Consequently, $\left(\boldsymbol{\Omega} \rho \otimes I d_{C}\right) D=\widetilde{D}\left(\boldsymbol{\Omega} \rho \otimes I d_{C}\right)$, since $\boldsymbol{\Omega} \rho \otimes I d_{C}$ is a morphism of (free) right $\boldsymbol{\Omega} C$-modules. Thus $D^{2}=0$ implies that $\widetilde{D}^{2}=0$.

Let $\sigma: C @>>>\otimes C$ be the right inverse to $\rho$, i.e., $\sigma(c)=1 \otimes c$ for all $c \in C$. Note that $\sigma$ is a morphism of cochain coalgebras. Furthermore, the equality $(\dagger)$ implies that

$$
D(1 \otimes c)-\left(\boldsymbol{\Omega} \sigma \otimes I d_{C}\right) \widetilde{D}(1 \otimes c) \in \operatorname{ker}\left(\boldsymbol{\Omega} \rho \otimes I d_{C}\right)=\boldsymbol{\Omega}\left(C^{+} \otimes C\right) \otimes C
$$

for all $c \in C$.

Let us simplify notation by writing $\tilde{\mu}(w \otimes z)=w \cdot z$ for all $w, z \in \boldsymbol{\Omega} C \otimes C$. The definition of $\tilde{\mu}$ is chosen so that

$$
\left(s^{-1} a \otimes 1\right) \cdot(1 \otimes c)=\left(\boldsymbol{\Omega} \rho \otimes I d_{C}\right)\left(\left(s^{-1}(1 \otimes a) \otimes 1\right) \cdot(1 \otimes c)\right)
$$

for all $a, c \in C$, which leads easily to the more general identity that

$$
w \cdot z=\left(\boldsymbol{\Omega} \rho \otimes I d_{C}\right)\left(\left(\boldsymbol{\Omega} \sigma \otimes I d_{C}\right)(w) \cdot\left(\boldsymbol{\Omega} \sigma \otimes I d_{C}\right)(z)\right)
$$

for all $w, z \in \mathbf{\Omega} C \otimes C$. Thus

$$
\begin{aligned}
\widetilde{D}\left(\left(s^{-1} a \otimes 1\right) \cdot(1 \otimes c)\right) \\
=\left(\boldsymbol{\Omega} \rho \otimes I d_{C}\right) D\left(\left(s^{-1}(1 \otimes a) \otimes 1\right) \cdot(1 \otimes c)\right) \\
=\left(\boldsymbol{\Omega} \rho \otimes I d_{C}\right)\left(\left(d s^{-1}(1 \otimes a) \otimes 1\right) \cdot(1 \otimes c)\right. \\
\left.\quad+(-1)^{a+1}\left(s^{-1}(1 \otimes a) \otimes 1\right) \cdot D(1 \otimes c)\right) \\
=\left(\boldsymbol{\Omega} \rho \otimes I d_{C}\right)\left(\left(\boldsymbol{\Omega} \sigma \otimes I d_{C}\right)\left(d s^{-1} a \otimes 1\right) \cdot\left(\boldsymbol{\Omega} \sigma \otimes I d_{C}\right)(1 \otimes c)\right. \\
\left.\quad+(-1)^{a+1}\left(\boldsymbol{\Omega} \sigma \otimes I d_{C}\right)\left(s^{-1} a \otimes 1\right) \cdot D(1 \otimes c)\right) \\
=\left(\boldsymbol{\Omega} \rho \otimes I d_{C}\right)\left(\left(\boldsymbol{\Omega} \sigma \otimes I d_{C}\right)\left(d s^{-1} a \otimes 1\right) \cdot\left(\boldsymbol{\Omega} \sigma \otimes I d_{C}\right)(1 \otimes c)\right. \\
\left.\quad+(-1)^{a+1}\left(\boldsymbol{\Omega} \sigma \otimes I d_{C}\right)\left(s^{-1} a \otimes 1\right) \cdot\left(\boldsymbol{\Omega} \sigma \otimes I d_{C}\right) \widetilde{D}(1 \otimes c)\right) \\
=\left(d s^{-1} a \otimes 1\right) \cdot(1 \otimes c)+(-1)^{a+1}\left(s^{-1} a \otimes 1\right) \cdot \widetilde{D}(1 \otimes c),
\end{aligned}
$$


where the second-to-last equality is due to $(\ddagger)$, since $\operatorname{ker}\left(\boldsymbol{\Omega} \rho \otimes I d_{C}\right)$ is obviously a two-sided $\Omega(C \otimes C)$-module.

We have thus established that $\widetilde{D}$ is a dervation with respect to $\tilde{\mu}$.

Proposition 2.2. Let $\bar{\gamma}: \Omega C \odot C @>>>C^{*}\left(P_{*} Y ; R\right)$ be the graded $R$-module morphism defined by

$$
\bar{\gamma}(\omega \otimes c)=(-1)^{\omega c} \bar{\imath}^{\sharp} \beta(1 \otimes c) \cdot \bar{q}^{\sharp} \gamma(\omega)
$$

for all $\omega \in \mathbf{\Omega} C$ and all $c \in C$. Then $\bar{\gamma}$ is a quasi-isomorphism of right $\boldsymbol{\Omega} C$-modules inducing an algebra homomorphism in cohomology.

Remark. That $\bar{\gamma}$ is a quasi-isomorphism implies immediately that it induces an algebra homomorphism in cohomology, since $H^{*}\left(P_{*} Y ; R\right)=R$, concentrated in degree 0 .

Proof. We begin by verifying that $\bar{\gamma}$ is a differential map. Note first that $\bar{\gamma}$ is a morphism of right $\Omega C$-modules, i.e., it is clear that

$$
\bar{\gamma}\left((\omega \otimes c)\left(\omega^{\prime} \otimes 1\right)\right)=\bar{\gamma}(\omega \otimes c) \cdot \bar{q}^{\sharp} \gamma\left(\omega^{\prime}\right)
$$

for all $\omega, \omega^{\prime} \in \Omega C$ and $c \in C$.

Next observe that the following cubic diagram commutes.

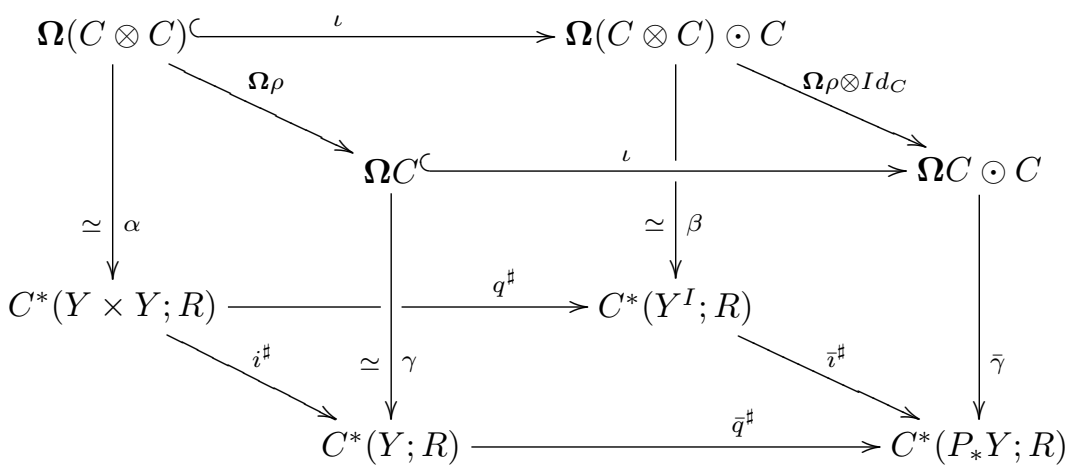

The only face for which the commutativity is not immediate is the right-hand vertical face, where for all $\omega \in \boldsymbol{\Omega}(C \otimes C)$ and for all $c \in C$

$$
\begin{aligned}
\bar{\gamma}\left(\boldsymbol{\Omega} \rho \otimes I d_{C}\right)(\omega \otimes c) & =\bar{\gamma}(\boldsymbol{\Omega} \rho(\omega) \otimes c) \\
& =(-1)^{\omega c} \bar{\imath}^{\sharp} \beta(1 \otimes c) \cdot \bar{q}^{\sharp} \gamma \boldsymbol{\Omega} \rho(\omega) \\
& =(-1)^{\omega c} \bar{\imath}^{\sharp} \beta(1 \otimes c) \cdot \bar{\imath}^{\sharp} \beta \iota(\omega) \\
& =\bar{\imath}^{\sharp} \beta(\omega \otimes c) \quad \text { since } \beta \text { is a map of right } \boldsymbol{\Omega}(C \otimes C) \text {-modules. }
\end{aligned}
$$

Thus, in particular, $\bar{\imath}^{\sharp} \beta D(1 \otimes c)=\bar{\gamma} \widetilde{D}(1 \otimes c)$ for all $c \in C$. Consequently, if $\delta$ is 
the differential on $C^{*}\left(P_{*} Y ; R\right)$, then

$$
\begin{aligned}
\delta \bar{\gamma}(\omega \otimes c) & =(-1)^{\omega c} \delta \bar{\imath}^{\sharp} \beta(1 \otimes c) \cdot \bar{q}^{\sharp} \gamma(\omega)+(-1)^{(\omega+1) c} \bar{\imath}^{\sharp} \beta(1 \otimes c) \cdot \delta \bar{q}^{\sharp} \gamma(\omega) \\
& =(-1)^{\omega c} \bar{\imath}^{\sharp} \beta D(1 \otimes c) \cdot \bar{q}^{\sharp} \gamma(\omega)+(-1)^{(\omega+1) c} \bar{\imath}^{\sharp} \beta(1 \otimes c) \cdot \bar{q}^{\sharp} \gamma(d \omega) \\
& =(-1)^{\omega c} \bar{\gamma} \widetilde{D}(1 \otimes c) \cdot \bar{q}^{\sharp} \gamma(\omega)+\bar{\gamma}(d \omega \otimes c) \\
& =\bar{\gamma}\left((-1)^{\omega c} \widetilde{D}(1 \otimes c) \cdot(\omega \otimes 1)+d \omega \otimes c\right)
\end{aligned}
$$

for all $\omega \in \boldsymbol{\Omega} C$ and $c \in C$, i.e., $\delta \bar{\gamma}=\bar{\gamma} \widetilde{D}$.

A straightforward Eilenberg-Moore spectral sequence argument, similar to that given in the proof of Theorem 1.4 in [5], now implies that $\bar{\gamma}$ is a quasi-isomorphism.

In summary, we have constucted a quasi- $R$-model of $\bar{q}$

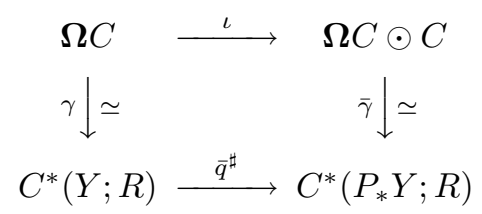

in which $\bar{\gamma}$ is a morphism of right $\Omega C$-modules such that $H^{*} \bar{\gamma}$ is an algebra homomorphism, and all other morphisms are morphisms of cochain algebras. If we apply the definition of the differential $\widetilde{D}$ recursively, we obtain

$$
\begin{aligned}
\widetilde{D}(\omega \otimes c)= & d \omega \otimes c+(-1)^{\omega} \omega \otimes \partial c \\
& +(-1)^{\omega(c+1)} s^{-1} c \omega \otimes 1+\sum_{j \in J}(-1)^{\omega\left(c^{j}+1\right)} s^{-1} c^{j} \omega \otimes c_{j}
\end{aligned}
$$

for all $\omega \in \boldsymbol{\Omega} C$ and $c \in C$, where $\bar{\Delta}(c)=\sum_{j \in J} c_{j} \otimes c^{j}$. The complex $(\boldsymbol{\Omega} C \otimes C, \widetilde{D})$ underlying $\Omega C \odot C$ is therefore isomorphic to the acyclic cobar construction, which we have thus endowed with a product structure and a specific map into $C^{*}\left(P_{*} Y ; R\right)$.

\section{The homotopy fiber model}

In this section we explain how to construct a quasi- $R$-model of the homotopy fiber of continuous map that possesses a strict Hopf model. We present in section 3.1 a general, noncommutative construction, from which we derive in section 3.2 simpler, commutative models that apply in special cases.

\subsection{The noncommutative model}

Recall first that the homotopy fiber $F$ of a continuous map $f: X @>>>$ has the homotopy type of the pull-back of $f$ and of the based path space fibration $\bar{q}: P_{*} Y @>>>Y$, as in the diagram below.

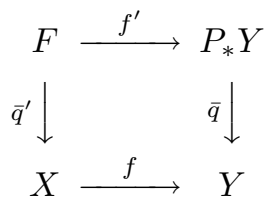


It is thus natural that our model of $F$ be a push-out of the model (2.6) of $\bar{q}$ and of an appropriate model of $f$, as we describe below.

Observe furthermore that the homotopy fiber of any continuous map $f$ has the same homotopy type as the homotopy fiber of the inclusion of $X$ into the mapping cylinder of $f$. We may therefore assume that the map of which we are computing the homotopy fiber is injective. If $X$ and $Y$ are CW-complexes, this argument permits us to assume that $f$ is an inclusion of a subcomplex into a larger complex.

Henceforth in this section, suppose that $f: X @>>>Y$ is an injective continuous map that possesses a strict Hopf model

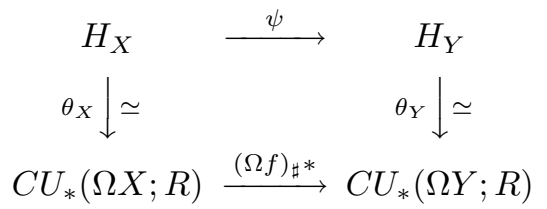

from which we derive, as in section 1 , a strict cochain model

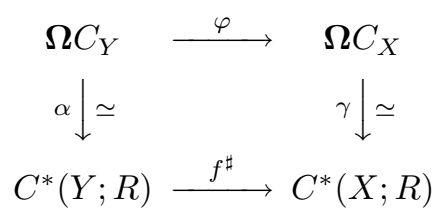

where $C_{X}$ and $C_{Y}$ are the $R$-duals of $H_{X}$ and $H_{Y}$, as usual. Let

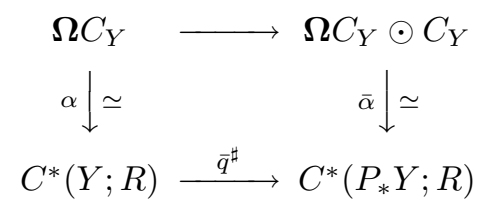

be the quasi- $R$-model of the based path fibration constructed in section 2 . Let $F$ denote the homotopy fiber of $f$.

We define now our candidate for a model of $F$, as a "push-out" of the models (3.2) and (3.3). Let $\partial_{X}$ and $\partial_{Y}$ denote the differentials on $C_{X}$ and $C_{Y}, d_{X}$ and $d_{Y}$ the differentials on $\Omega C_{X}$ and $\Omega C_{Y}$, and $\Delta_{X}$ and $\Delta_{Y}$ the coproducts on $C_{X}$ and $C_{Y}$.

Definition 1. Definition of $\Omega C_{X} \odot C_{Y}$ Let $\Omega C_{X} \odot C_{Y}$ denote the graded $R$-module $\Omega C_{X} \otimes C_{Y}$ endowed with an associative product

$$
\mu^{\prime}:\left(\boldsymbol{\Omega} C_{X} \otimes C_{Y}\right) \otimes\left(\Omega C_{X} \otimes C_{Y}\right) @>>>\Omega C_{X} \otimes C_{Y}
$$

and a degree +1 endomorphism extending $d_{X}$

$$
D^{\prime}: \Omega C_{X} \otimes C_{Y} @>>>\Omega C_{X} \otimes C_{Y}
$$

determined by the conditions that for all $a \in C_{X}$ and $c, c^{\prime} \in C_{Y^{*}}$ with $\bar{\Delta}_{Y} *(c)=$ $\sum_{j \in J} c_{j} \otimes c^{j}$ :

1.

$$
\mu^{\prime}\left((1 \otimes c) \otimes\left(s^{-1} a \otimes 1\right)\right)=(-1)^{c(a+1)} s^{-1} a \otimes c ;
$$


2.

$$
\begin{aligned}
\mu^{\prime}\left(\left(s^{-1} a \otimes 1\right) \otimes(1 \otimes c)\right)= & s^{-1} a \otimes c+s^{-1}\left(a \cdot \psi^{\star}(c)\right) \otimes 1 \\
& +\sum_{j \in J}(-1)^{c_{j} c^{j}} s^{-1}\left(a \cdot \psi^{\star}\left(c^{j}\right)\right) \otimes c_{j} ;
\end{aligned}
$$

3.

$$
\mu^{\prime}\left((1 \otimes c) \otimes\left(1 \otimes c^{\prime}\right)\right)=1 \otimes c c^{\prime}
$$

4. the perturbation of $1 \otimes \partial_{Y}$ is defined by

$$
D^{\prime}(1 \otimes c)=1 \otimes \partial_{Y} c-s^{-1} \psi^{\star}(c)-\sum_{j \in J}(-1)^{c_{j} c^{j}} s^{-1} \psi^{\star}\left(c^{j}\right) \otimes c_{j} ;
$$

5. $D^{\prime}$ is defined to commute with the right action of $\Omega C_{X}$, i.e.,

$$
D^{\prime}\left(s^{-1} a \otimes c\right)=d_{X} s^{-1} a \otimes c+(-1)^{c(a+1)}\left(D^{\prime}(1 \otimes c)\right) \cdot\left(s^{-1} a \otimes 1\right) .
$$

Proposition 3.1. As defined above, $\boldsymbol{\Omega} C_{X} \odot C_{Y}=\left(\boldsymbol{\Omega} C_{X} \otimes C_{Y}, D^{\prime}, \mu^{\prime}\right)$ is an associative cochain algebra.

Proof. Notice that $D^{\prime}$ is defined precisely so that

$$
D^{\prime}(1 \otimes c)=\left(\varphi \otimes I d_{C_{Y}}\right) \widetilde{D}(1 \otimes c)
$$

for all $c$, which implies that $D^{\prime}\left(\varphi \otimes I d_{C_{Y *}}\right)=\left(\varphi \otimes I d_{C_{Y *}}\right) \widetilde{D}$, since $\varphi \otimes I d_{C_{Y *}}$ is a morphism of right $\Omega C_{Y} *$-modules. Thus $\widetilde{D}^{2}=0$ implies that $D^{\prime 2}=0$.

The proof that $D^{\prime}$ is a derivation with respect to $\mu^{\prime}$ follows easily from direct calculations based on their definitions. We spare the reader the details of the computation, mentioning only that the identities

$$
\left(\partial_{Y} \otimes 1+1 \otimes \partial_{Y}\right) \Delta_{Y}=\Delta_{Y} \partial_{Y} \quad \text { and } \quad\left(\Delta_{Y} \otimes 1\right) \Delta_{Y}=\left(1 \otimes \Delta_{Y}\right) \Delta_{Y}
$$

and the fact that $\psi^{\star}$ and the product in $C_{X}$ are maps of coalgebras are all essential to its success.

The next proposition states that the cochain algebra constructed above really is a model of $F$, although in a somewhat weaker sense than the other models we have constructed thus far in this article.

In what follows, given two morphisms of cochain algebras $A @>>>B$ and $A @>>>B^{\prime}$, let $\left\{E_{r}, d_{r}\right\}$ denote the Eilenberg-Moore spectral sequence with

$$
E_{2}^{*, *} \cong \operatorname{Tor}_{H^{*}(A)}^{* * *}\left(H^{*}(B), H^{*}\left(B^{\prime}\right)\right),
$$

converging to $\operatorname{Tor}_{A}^{* * *}\left(B, B^{\prime}\right)$. If $g: X @>>>B$ is a continuous map and $p: E @>>>$ $B$ is a fibration, then let $\theta_{p, g^{*}}: \operatorname{Tor}_{C^{*}(B ; R)}^{* * *}\left(C^{*}(E ; R), C^{*}(X ; R)\right) @>\cong>>H^{*}(E \underset{B}{\longrightarrow}$ $\times X ; R)$ denote the canonical isomorphism of algebras. Recall that the EilenbergMoore spectral sequence of two cochain algebra morphisms is a spectral sequence of algebras. For further details, we refer the reader to chapters 7 and 8 in [12].

Proposition 3.2. Let $\zeta: \Omega C_{X} \odot C_{Y} @>>>C^{*}(F ; R)$ be the graded R-module morphism defined by

$$
\zeta(\omega \otimes c)=(-1)^{\omega c} f^{\prime \sharp} \bar{\alpha}(1 \otimes c) \cdot q^{\sharp \sharp} \gamma(\omega)
$$


for all $\omega \in \boldsymbol{\Omega} C_{X^{*}}$ and all $c \in C_{Y^{*}}$. Then $\zeta$ is a quasi-isomorphism of right $\Omega C_{X} *$-modules. More precisely, $H^{*} \zeta=\theta_{\bar{q}, f} \circ \operatorname{Tor}_{\alpha}(\bar{\alpha}, \gamma)$, and the bigraded morphism $E_{0}\left(\operatorname{Tor}_{\alpha}(\bar{\alpha}, \gamma)\right)$ associated to $\operatorname{Tor}_{\alpha}(\bar{\alpha}, \gamma)$ is an isomorphism of algebras. Here, $E_{0}$ refers to the filtration induced on the Tor's by the usual Eilenberg-Moore filtration.*

Remark. While it would be preferable for $H^{*} \zeta$ itself, rather than its associated bigraded morphism, to be an algebra morphism, we were unable to prove this stronger result and even doubt that it is true in general. However, as we explain at the end of the proof of Proposition 3.2, there are special circumstances under which we can show that $H^{*} \zeta$ is an algebra morphism, e.g., when $f$ admits a retraction or when $\bar{\alpha}$ can be chosen so that $1 \otimes \operatorname{ker} \psi^{\star} \subseteq \operatorname{ker} f^{\prime \sharp} \bar{\alpha}$. Furthermore, in some situations the fact that the associated bigraded morphism preserves the product implies that the same holds for $H^{*} \zeta$, for degree reasons. We present examples of such calculations in section 4 .

Proof. The proof that $\zeta$ is a quasi-isomorphism follows an outline identical to that of Proposition 2.2. We observe first that $\zeta$ is a morphism of $\Omega C_{X}$-modules, i.e., that

$$
\zeta\left((\omega \otimes c) \cdot\left(\omega^{\prime} \otimes 1\right)\right)=\zeta(\omega \otimes c) \cdot q^{\prime \sharp} \gamma(\omega)
$$

for all $\omega, \omega^{\prime} \in \Omega C_{X}$ and $c \in C_{Y}$. We can then establish easily that the cubic diagram

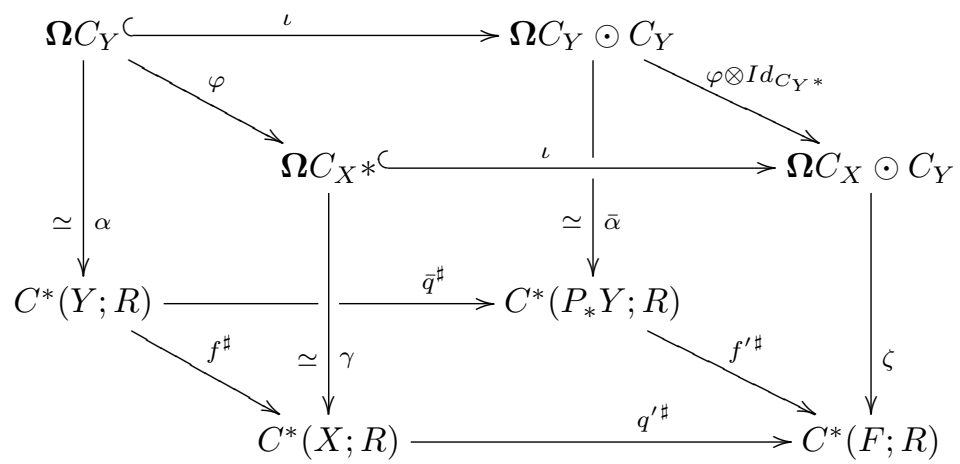

commutes, enabling us to apply the usual Eilenberg-Moore spectral sequence argument to establish that $\zeta$ is a quasi-isomorphism.

In fact, it is clear that $H^{*} \zeta$ is exactly the composition

$$
\begin{gathered}
\operatorname{Tor}_{\Omega C_{Y}}\left(\Omega C \odot C, C_{X}\right) @>\operatorname{Tor}_{\alpha}(\bar{\alpha}, \gamma)>\cong>\operatorname{Tor}_{C^{*} Y}\left(C^{*} P_{*} Y, C^{*} X\right) @> \\
\theta_{\bar{q}, f *>}>>H^{*} F,
\end{gathered}
$$

where we have supressed the coefficients from the notation. The first morphism, $\operatorname{Tor}_{\alpha}(\bar{\alpha}, \gamma)$, is an isomorphism because $\operatorname{Tor}_{H^{*} \alpha}\left(H^{*} \bar{\alpha}, H^{*} \gamma\right)$ is an isomorphism.

To see that the induced morphism on the $E_{\infty}$-terms respects the algebra structure, we establish first that $\varphi \otimes I d_{C}$ does so. Let $a \in C_{X}$ and $c \in C_{Y}$, with $\bar{\Delta}(c)=\sum_{j \in J} c_{j} \otimes c^{j}$. Suppose that $b \in\left(\psi^{\star}\right)^{-1}(a)$. Then, since $\psi^{\star}$ is a morphism of 
algebras,

$$
\begin{aligned}
\left(\varphi \otimes I d_{C_{Y}}\right) & \left(\left(s^{-1} b \otimes 1\right) \cdot(1 \otimes c)\right) \\
& =\left(\varphi \otimes I d_{C_{Y}}\right)\left(s^{-1} b \otimes c+s^{-1}(b \cdot c) \otimes 1+\sum_{j \in J}(-1)^{c_{j} c^{j}} s^{-1}\left(b \cdot c^{j}\right) \otimes c_{j}\right) \\
& =s^{-1} a \otimes c+s^{-1}\left(a \cdot \psi^{\star}(c)\right) \otimes 1+\sum_{j \in J}(-1)^{c_{j} c^{j}} s^{-1}\left(a \cdot \psi^{\star}\left(c^{j}\right)\right) \otimes c_{j} \\
& =\left(s^{-1} a \otimes 1\right)(1 \otimes c) .
\end{aligned}
$$

This result generalizes by a simple inductive argument to show that if $\xi \in \varphi^{-1}(\omega)$ and $\xi^{\prime} \in \varphi^{-1}\left(\omega^{\prime}\right)$, for some $\omega, \omega^{\prime} \in \Omega C_{X}$, then

$$
\left(\varphi \otimes I d_{C_{Y}}\right)\left((\xi \otimes c) \cdot\left(\xi^{\prime} \otimes c^{\prime}\right)\right)=(\omega \otimes c) \cdot\left(\omega^{\prime} \otimes c^{\prime}\right)
$$

for all $c, c^{\prime} \in C_{Y}$. In other words, $\varphi \otimes I d_{C_{Y}}$ is a morphism of algebras.

Next consider the cubic diagram

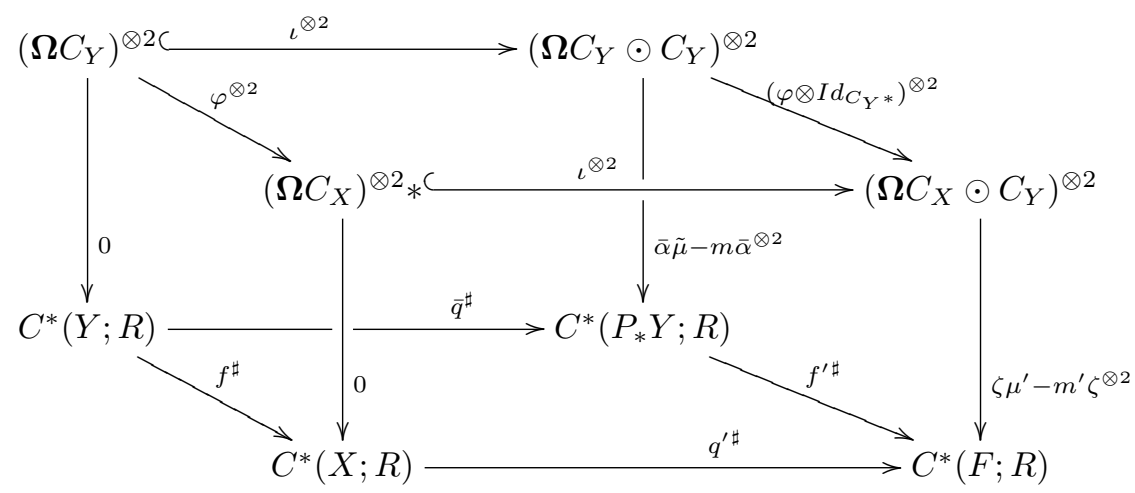

where $m$ and $m^{\prime}$ denote the products on $C^{*}\left(P_{*} Y ; R\right)$ and $C^{*}(F ; R)$, respectively. The right-hand face of the cube commutes because $\varphi \otimes I d_{C_{Y}}$ is a morphism of algebras. The commutativity of the other faces is immediate. Since the cube commutes,

$$
H^{*}\left(\zeta \mu^{\prime}-m^{\prime} \zeta^{\otimes 2}\right)=\theta_{p, f} \circ \operatorname{Tor}_{0}\left(\bar{\alpha} \tilde{\mu}-m \bar{\alpha}^{\otimes 2}, 0\right) .
$$

The morphism $\operatorname{Tor}_{0}\left(H^{*}\left(\bar{\alpha} \tilde{\mu}-m \bar{\alpha}^{\otimes 2}\right), 0\right)$ on the $E_{2}$-terms of the Eilenberg-Moore spectral sequence is zero, since $H^{*}\left(\bar{\alpha} \tilde{\mu}-m \bar{\alpha}^{\otimes 2}\right)=0$. The morphism induced on the $E_{\infty}$-terms is therefore also zero, which means that

$$
E_{0}\left(H^{*}\left(\zeta \mu^{\prime}\right)\right)=E_{0}\left(H^{*}\left(m^{\prime} \zeta^{\otimes 2}\right)\right)
$$

i.e., $E_{0}\left(H^{*} \zeta\right)$ is a morphism of algebras.

We now present circumstances under which we can establish that $H^{*} \zeta$ itself is an algebra morphism. Since $f$ is assumed to be injective, we can assume that the strict Hopf model (3.1) is injective. The dual map $\psi^{\star}$ is then surjective, so there is a section $\sigma: C_{X} @>>>C_{Y}$ of $\psi^{\star}$ as morphisms of graded $R$-modules, since all complexes are $R$-free. Let $S: \Omega C_{X} \odot C_{Y} @>>>\Omega C_{X} \odot C_{Y}$ be the morphism of graded $R$-modules of degree -1 given by

$$
S\left(s^{-1} a_{1} \cdots s^{-1} a_{n} \otimes 1\right)=(-1)^{\varepsilon} s^{-1} a_{2} \cdots s^{-1} a_{n} \otimes \sigma\left(a_{1}\right),
$$


where $\varepsilon=1+\left|a_{1}\right| \cdot \sum_{i=2}^{n}\left(\left|a_{i}\right|+1\right)$, and

$$
S(\omega \otimes c)=0 \quad \text { whenever }|c|>0 .
$$

Straightforward calculations then lead to

$$
\left(D^{\prime} S+S D^{\prime}\right)(\omega \otimes c)=\omega \otimes \sigma \psi^{\star}(c)
$$

and to

$$
\begin{aligned}
\left(D^{\prime} S+S D^{\prime}\right)\left(s^{-1} a_{1} \cdots s^{-1} a_{n} \otimes\right. & 1) \\
= & s^{-1} a_{1} \cdots s^{-1} a_{n} \otimes 1 \\
& \pm s^{-1} a_{2} \cdots s^{-1} a_{n} \otimes\left(\partial_{Y} \sigma-\sigma \partial_{X}\right)\left(a_{1}\right) \\
& +\sum_{j \in J} \pm s^{-1} a_{1}^{j} s^{-1} a_{2} \cdots s^{-1} a_{n} \otimes\left(\sigma\left(a_{1}\right)_{j}-\sigma\left(a_{1 j}\right)\right)
\end{aligned}
$$

where we use our usual notation for the summands of the reduced coproduct, and we have taken the liberty of replacing complicated sign exponents by \pm , for the sake of legibility.

Let $\delta^{\prime}$ denote the differential on $C^{*}(F ; R)$. Let

$$
G=\left(\zeta \mu^{\prime}-m^{\prime}(\zeta \otimes \zeta)\right)(S \otimes I d): \Omega C_{X} \odot C_{Y} \otimes \Omega C_{X} \odot C_{Y} @>>>C^{*}(F ; R)
$$

so that if $|c|>0$, then

$$
\begin{aligned}
\delta^{\prime} G & +G\left(D^{\prime} \otimes I d+I d \otimes D^{\prime}\right)\left(\omega \otimes c \otimes \omega^{\prime} \otimes c^{\prime}\right) \\
& = \pm \zeta\left(1 \otimes \sigma \psi^{\star}(c)\right) \cdot\left(\zeta\left((\omega \otimes 1) \cdot\left(1 \otimes c^{\prime}\right)\right)-\zeta(\omega \otimes 1) \cdot \zeta\left(1 \otimes c^{\prime}\right)\right) \cdot \zeta\left(\omega^{\prime} \otimes 1\right) .
\end{aligned}
$$

Furthermore, if $\omega=s^{-1} a_{1} \cdots s^{-1} a_{n}$ and $\bar{\omega}=s^{-1} a_{2} \cdots s^{-1} a_{n}$, then

$$
\begin{aligned}
\delta^{\prime} G+ & G\left(D^{\prime} \otimes I d+I d \otimes D^{\prime}\right)\left(\omega \otimes 1 \otimes \omega^{\prime} \otimes c^{\prime}\right) \\
= & \left(\zeta\left((\omega \otimes 1) \cdot\left(1 \otimes c^{\prime}\right)\right)-\zeta(\omega \otimes 1) \cdot \zeta\left(1 \otimes c^{\prime}\right)\right) \cdot \zeta\left(\omega^{\prime} \otimes 1\right) \\
+ & \zeta\left(1 \otimes\left(\partial_{Y} \sigma-\sigma \partial_{X}\right)\left(a_{1}\right)\right)\left(\zeta\left((\bar{\omega} \otimes 1) \cdot\left(1 \otimes c^{\prime}\right)\right)\right. \\
& \left.-\zeta(\bar{\omega} \otimes 1) \cdot \zeta\left(1 \otimes c^{\prime}\right)\right) \cdot \zeta\left(\omega^{\prime} \otimes 1\right) \\
+ & \left(1 \otimes \sigma\left(a_{1}\right)_{j}-\sigma\left(a_{1 j}\right)\right)\left(\zeta\left(\left(s^{-1} a_{1}^{j} \bar{\omega} \otimes 1\right) \cdot\left(1 \otimes c^{\prime}\right)\right)\right. \\
& \left.-\zeta\left(s^{-1} a_{1}^{j} \bar{\omega} \otimes 1\right) \cdot \zeta\left(1 \otimes c^{\prime}\right)\right) \cdot \zeta\left(\omega^{\prime} \otimes 1\right) .
\end{aligned}
$$

If $1 \otimes \operatorname{ker} \psi^{\star} \subseteq \operatorname{ker} f^{\prime \sharp} \bar{\alpha}$, then $\zeta\left(1 \otimes \sigma \psi^{\star}(c)\right)=\zeta(1 \otimes c)$ for all $c \in C_{Y}$, and

$$
\zeta\left(1 \otimes\left(\partial_{Y} \sigma-\sigma \partial_{X}\right)(a)\right)=0=\zeta\left(1 \otimes \sigma\left(a_{1}\right)_{j}-\sigma\left(a_{j}\right)\right)
$$

for all $a \in C_{X}$. Therefore

$$
\delta^{\prime} G+G\left(D^{\prime} \otimes I d+I d \otimes D^{\prime}\right)=\zeta \mu^{\prime}-m^{\prime}(\zeta \otimes \zeta),
$$


so that $H^{*} \zeta$ is an algebra morphism.

Now suppose that $f$ admits a retraction. Then $\psi$ can be defined so that it admits a retraction as a morphism of chain algebras, i.e., so that $\psi^{\star}$ admits a section $\sigma$ that is a morphism of cochain coalgebras. The algebra morphism $\boldsymbol{\Omega} \sigma$ is then a section of $\varphi$.

An easy chase in the commuting cube (3.4) shows that $\zeta=f^{\prime \sharp} \bar{\alpha}\left(\boldsymbol{\Omega} \sigma \otimes I d_{C_{Y}}\right)$. Let $\mu_{X}$ and $\mu_{Y}$ denote the products on $\Omega C_{X}$ and $\Omega C_{Y}$. Another chase in (3.4) establishes that

$$
\operatorname{Im}\left(\mu_{Y}\left(\boldsymbol{\Omega} \sigma \otimes I d_{\boldsymbol{\Omega} C_{Y} *}\right)-\boldsymbol{\Omega} \sigma \mu_{X}\left(I d_{\boldsymbol{\Omega} C_{X}} \otimes \varphi\right)\right) \subseteq \operatorname{ker} f^{\prime \sharp} \bar{\alpha} \iota,
$$

which implies that

$$
f^{\prime \sharp} \bar{\alpha} \tilde{\mu}\left(\boldsymbol{\Omega} \sigma \otimes I d_{C_{Y}}\right)^{\otimes 2}=f^{\prime \sharp} \bar{\alpha}\left(\boldsymbol{\Omega} \sigma \otimes I d_{C_{Y}}\right) \mu^{\prime} .
$$

Since $\Omega C \odot C$ is $R$-free and acyclic, there is a cochain homotopy

$$
K: \Omega C \odot C \otimes \Omega C \odot C @>>>C^{*}\left(P_{*} Y ; R\right)
$$

satisfying

$$
\delta K+K(\widetilde{D} \otimes I d+I d \otimes \widetilde{D})=\bar{\alpha} \tilde{\mu}-m \bar{\alpha}^{\otimes 2} .
$$

Let $K^{\prime}=f^{\prime \sharp} K(\boldsymbol{\Omega} \sigma \otimes I d)^{\otimes 2}$. Then

$$
\begin{aligned}
\delta^{\prime} K^{\prime}+K^{\prime}\left(D^{\prime} \otimes I d+I d \otimes D^{\prime}\right)= & f^{\prime \sharp} \bar{\alpha} \tilde{\mu}\left(\boldsymbol{\Omega} \sigma \otimes I d_{C_{Y}}\right)^{\otimes 2} \\
& -f^{\prime \sharp} m(\bar{\alpha}(\boldsymbol{\Omega} \sigma \otimes I d))^{\otimes 2} \\
= & f^{\prime \sharp} \bar{\alpha}\left(\boldsymbol{\Omega} \sigma \otimes I d_{C_{Y}}\right) \mu^{\prime} \\
& \left.-m^{\prime} f^{\prime \sharp} \bar{\alpha}(\boldsymbol{\Omega} \sigma \otimes I d)\right)^{\otimes 2} \\
= & \zeta \mu^{\prime}-m^{\prime} \zeta^{\otimes 2} .
\end{aligned}
$$

Thus, $H^{*} \zeta$ is an algebra morphism.

In summary, we have constructed a commuting diagram of cochain algebras

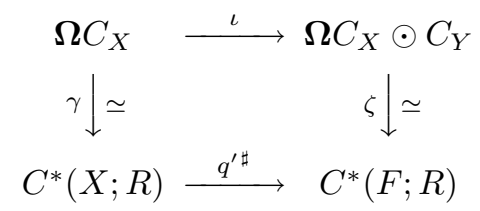

in which all morphisms except $\zeta$ are algebra morphisms. The bigraded morphism associated to $H^{*} \zeta$ is an algebra morphism, and we have mentioned special cases in which $H^{*} \zeta$ itself is an algebra morphism.

\subsection{Commutative models}

When $X$ and $Y$ belong to certain large and interesting classes of spaces, we can simplify the model (3.6) considerably, leading to greater ease of computation. The key to the simplifications we present in this section is the following lemma, proved in $[7]$. 
Lemma 3.3 ([7]). Let $A, B$ and $A \odot B=(A \otimes B, \mu, D)$ be cochain algebras over $R$ such that $A$ is a cochain subalgebra of $A \odot B,(1 \otimes b) \cdot(a \otimes 1)=(-1)^{a b} a \otimes b$ for all $a \in A$ and $b \in B$, and $B$ is isomorphic as a cochain algebra to the quotient of $A \odot B$ by the ideal $A^{>0} \otimes B$. Let $\eta: A @>>>A^{\prime}$ be a surjective quasi-isomorphism of cochain algebras such that

1. $(a \otimes 1) \cdot(1 \otimes b)-a \otimes b \in \operatorname{ker} \eta \otimes B$ for all $a \in A, b \in B$ and

2. $(1 \otimes b) \cdot\left(1 \otimes b^{\prime}\right)-1 \otimes b b^{\prime} \in \operatorname{ker} \eta \otimes B$ for all $b, b^{\prime} \in B$.

Endow the tensor product $A^{\prime} \otimes B$ of graded $R$-modules with the usual multiplication of a tensor product of algebras, and define $D^{\prime}: A^{\prime} \otimes B @>>A^{\prime} \otimes B$ by

$$
D\left(a^{\prime} \otimes b\right)=d^{\prime} a^{\prime} \otimes b+(-1)^{(b+1) a^{\prime}}\left(\eta \otimes I d_{B}\right) D(1 \otimes b) \cdot\left(a^{\prime} \otimes 1\right)
$$

for all $a^{\prime} \in A^{\prime}, b \in B$, where $d^{\prime}$ is the differential on $A^{\prime}$. Then $\eta \otimes I d_{B}: A \odot B @>>>$ $\left(A^{\prime} \otimes B, D^{\prime}\right)$ is a quasi-isomorphism of cochain algebras.

A cochain algebra $A \odot B$ satisfying the conditions in the lemma above possesses a natural decreasing, differential, multiplicative filtration $\left\{\mathcal{F}^{k}(A \odot B)\right\}_{k \geqslant 0}$ defined by

$$
\mathcal{F}^{k}(A \odot B)=A^{\geqslant k} \odot B .
$$

This filtration induces a spectral sequence of algebras converging to $H^{*}(A \odot B)$ and, in particular, a filtration of $H^{*}(A \odot B)$. We call the bigraded algebra associated to $H^{*}(A \odot B)$ by the induced filtration its natural associated bigraded algebra.

The first simplification of the model (3.5) applies to $p$-Anick spaces, among others. Its definition involves the enveloping algebra functor $U$ from the category of chain Lie algebras to that of chain Hopf algebras. Recall that $U L$ is a cocommutative, coassociative chain Hopf algebra for any chain Lie algebra $L$.

The definition of the model below also makes use of the Cartan-ChevalleyEilenberg cochain algebra $\mathcal{C}^{*}(L)$ of a chain Lie algebra $L$, over a subring of $\mathbb{Q}$ containing $\frac{1}{2}$. As an algebra $\mathcal{C}^{*}(L)$ is isomorphic to the free, graded commutative algebra $\Lambda s^{-1} L^{\star}$. The differential $d$ of $\mathcal{C}^{*}(L)$ is a sum $d_{1}+d_{2}$, where $d_{1}$ preserves wordlength and $d_{2}$ increases it by 1 . The two summands are specified by

$$
\begin{aligned}
\left\langle d_{1} \xi, s x\right\rangle & =(-1)^{\xi}\langle\xi, s \mathfrak{d} x\rangle \\
\left\langle d_{2} \xi, s x \cdot s y\right\rangle & =(-1)^{s y}\langle\xi, s[x, y]\rangle
\end{aligned}
$$

for all $\xi \in s^{-1} L^{\star}$ and $x, y \in L$, where $\langle$,$\rangle denotes evaluation and \mathfrak{d}$ is the differntial on $L$. For further details, see, for example, [11].

Corollary 3.4. Let $R$ be a subring of $\mathbb{Q}$ containing $\frac{1}{2}$. Suppose that there exists an injective morphism of finite-type chain Lie algebras $\mathfrak{f}: L_{X} @>>>L_{Y}$ that fits into the following strict Hopf model.

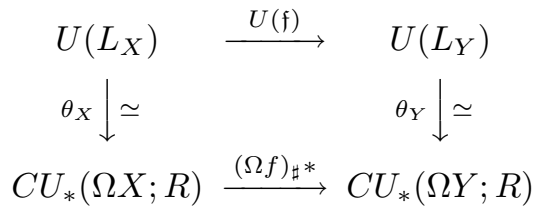


Let $C$ denote the $R$-dual of $U\left(L_{Y}\right)$. Then there exists a cochain algebra extension of $\mathcal{C}^{*}(L)$

$$
\mathcal{C}^{*}(L) \hookrightarrow\left(\Lambda s^{-1} L^{\star} \otimes C, D\right)=\mathcal{C}^{*}(L) \odot C
$$

such that the natural associated bigraded algebra of $H^{*}\left(\mathcal{C}^{*}(L) \odot C\right)$ is isomorphic to the (Eilenberg-Moore) associated bigraded algebra of $H^{*}(F ; R)$. The differential $D$ is defined by

$$
D(1 \otimes c)=1 \otimes \partial c-s^{-1}\left(i^{\star} \mathfrak{f}(c)\right)-\sum_{j \in J}(-1)^{c_{j} c^{j}} s^{-1}\left(i^{\star} \mathfrak{f}\left(c^{j}\right)\right) \otimes c_{j},
$$

where $\partial$ is the differential on $C, i: L_{X} \hookrightarrow U\left(L_{X}\right)$ is the natural inclusion, and $\bar{\Delta}(c)=\sum_{j \in J} c_{j} \otimes c^{j}$.

Existence. As explained in section 8 of $[\mathbf{2}]$ and mentioned above in section 1 , the hypotheses of Corollary 3.4 are satisfied whenever $X$ and $Y$ are CW-complexes with trivial $r$-skeleton and of dimension at most $r p$, where $p$ is the smallest prime that is not invertible in $R$.

Proof. Let $\mathfrak{p}: \Omega C @>>>\mathcal{C}^{*}(L)$ denote the cochain algebra map defined by $\mathfrak{p}\left(s^{-1} c\right)=s^{-1} i^{\star}(c)$ for all $c \in C$. As remarked in [11, 1.12], $\mathfrak{p}$ is a surjective quasiisomorphism. By an argument essentially identical to that in the proof of Theorem 1.1 in [7], we can verify that the hypotheses of Lemma 3.3 are satisfied by $\mathfrak{p}$ and the model (3.5), and thus obtain the model specified above.

In the next corollary, we impose a formality condition on $f$ in order to simplify (3.5). A space $X$ is said to be $R$-formal if there is a sequence of quasi-isomorphisms of cochain algebras

$$
C^{*}(X ; R) @>\simeq>>\bullet @<<<H^{*}(X ; R) .
$$

When $H^{*}(X ; R)$ is $R$-torsion free, it is easy to see that this condition is equivalent to the existence of a quasi-isomorphism of cochain algebras $\Omega H_{*}(X ; R) @>>>$ $\boldsymbol{\Omega} C_{*}(X ; R)$. We refer the reader to [4] for further details.

By analogy with the definition of formal spaces, we say that $f: X @>>>Y$ is $R$-formalizable if there is a strict Hopf model of the following form.

$$
\begin{array}{ccc}
\Omega H_{*}(X ; R) & \stackrel{\Omega H_{*} f}{\longrightarrow} & \Omega H_{*}(Y ; R) \\
\theta_{X} \downarrow \simeq & \theta_{Y} \downarrow \simeq \\
C U_{*}(\Omega X ; R) \stackrel{(\Omega f)_{\sharp^{*}}}{\longrightarrow} C U_{*}(\Omega Y ; R)
\end{array}
$$

Recall that the bar construction on an $R$-free algebra is a cofree coalgebra, which becomes a Hopf algebra when endowed with the shuffle product.

Proposition 3.5. Corollary 3.5 Let $R$ be a commutative, unitary ring. Suppose $f: X @>>>Y$ is R-formalizable, with strict Hopf model (3.6). Let $A=H^{*}(X ; R)$ and $B=H^{*}(Y ; R)$, and suppose they are $R$-torsion free. Then there exists a cochain algebra extension of $A$ by $\mathcal{B} B$

$$
A \hookrightarrow\left(A \otimes \perp s B^{+}, D\right)=A \odot \mathcal{B} B
$$


such that the natural associated bigraded algebra of $H^{*}(A \odot \mathcal{B} B)$ is isomorphic to the (Eilenberg-Moore) associated bigraded algebra of $H^{*}(F ; R)$. The differential $D$ is defined by

$$
D\left(1 \otimes s b_{1} \cdots s b_{n}\right)=1 \otimes \partial\left(s b_{1} \cdots s b_{n}\right)-(-1)^{\varepsilon} H^{*} f\left(b_{n}\right) \otimes s b_{1} \cdots s b_{n-1}
$$

where $\partial$ is the bar construction differential and $\varepsilon=\left(\left|b_{n}\right|+1\right) \cdot \sum_{i=1}^{n-1}\left(\left|b_{i}\right|+1\right)$.

Proof. Applying the construction in section 3.1 to the diagram (3.6), we obtain a model

$$
\zeta: \Omega \mathcal{B} A \odot \mathcal{B} B @>\simeq>>C^{*}(F ; R) .
$$

Consider the surjective quasi-isomorphism of cochain algebras $\eta: \Omega \mathcal{B} A @>\simeq>>A$ given by the adjunction. Just as in the proof of Theorem 1.2 in [7], the hypotheses of Lemma 3.3 are satisfied by $\eta$, so that we obtain the model defined in the statement of the corollary.

\section{Applications}

We present in this section two elementary examples of homotopy fiber models. We will devote a future article to further applications of the model, in particular to the study of homotopy fibers of cell attachments.

Given a basis $\left\{v_{1}, \ldots, v_{n}\right\}$ of a free graded $R$-module $V$, we will often abuse notation slightly and write $\perp\left(v_{1}, \ldots, v_{n}\right)$ instead of $\perp V$, and $T\left(v_{1}, \ldots, v_{n}\right)$ instead of $T V$.

In $\perp y$, we let

$$
y(n)= \begin{cases}n \text { times } \overbrace{y \cdots y}, & n>0 \\ 1, & n=1 .\end{cases}
$$

Recall that the shuffle product endows $\perp V$ with a Hopf algebra structure. Whenever we refer to "the algebra" $\perp V$ in this section, we mean the algebra structure given by the shuffle product.

\subsection{The homotopy fiber of the $p$ th power map}

Let $p$ be a prime, and let $f: S^{n} @>>>S^{n}$ denote the $p$ th power map on $S^{n}$, where $n \geqslant 2$, with homotopy fiber $S^{n}\{p\}$. We verify first that $f$ is $\mathbb{Z}$-formalisable. Let

$$
\theta: \Omega H_{*}\left(S^{n} ; \mathbb{Z}\right)=\left(T s^{-1} H_{+}\left(S^{n} ; \mathbb{Z}\right), 0\right) @>>>C U_{*}\left(\Omega S^{n} ; \mathbb{Z}\right)
$$

denote the unique cochain algebra map extending $\theta\left(s^{-1} u\right)=z$, where $u$ is a generator of $H_{n}\left(S^{n} ; \mathbb{Z}\right)$ and $z$ is a representative of the generator of $H_{n-1}\left(\Omega S^{n} ; \mathbb{Z}\right)$. Since $u$ is primitive and $\bar{\Delta}(z)$ is a boundary, $\theta$ is even a morphism of Hopf algebras up to homotopy. It is clear that $\theta$ is an quasi-isomorphism and that the diagram

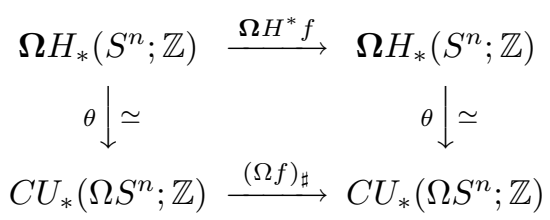


commutes, i.e., it is a strict Hopf model of $f$. Applying Corollary 3.4, we obtain a model

$$
\zeta:\left(\mathbb{Z}[v] /\left(v^{2}\right) \otimes \perp(s v), D\right) @>\simeq>>C^{*}(F ; \mathbb{Z})
$$

where $|v|=n, D(1 \otimes s v(m))=-(-1)^{(n+1)^{2}(m-1)} p \cdot v \otimes s v(m-1)$ for all $m \geqslant 1$ and $D(v \otimes s v(m))=0$ for all $m \geqslant 0$. Thus $H^{*}\left(\mathbb{Z}[v] /\left(v^{2}\right) \otimes \perp(s v), D\right)$ is the graded algebra with trivial product such that

$$
H^{k}\left(\mathbb{Z}[v] /\left(v^{2}\right) \otimes \perp(s v), D\right)= \begin{cases}\mathbb{Z} / p \mathbb{Z} & k=n+m(n+1), m \in \mathbb{N} \\ 0 & \text { else. }\end{cases}
$$

For degree reasons, the only possible product on this graded $\mathbb{Z}$-module is trivial, so $H^{*} \zeta$ is itself an algebra morphism.

If we tensor the above model with $\mathbb{F}_{p}$, the field of $p$ elements, we obtain a model for the homotopy fiber with mod $p$ coefficients. The differential on the $\mathbb{F}_{p}$-model is trivial, and we obtain algebra isomorphisms

$$
\begin{aligned}
H^{*}\left(\mathbb{F}_{p}[v] /\left(v^{2}\right) \otimes \perp(s v), 0\right) & \cong \mathbb{F}_{p}[v] /\left(v^{2}\right) \otimes \perp(s v) \\
& \cong H^{*}\left(S^{n} ; \mathbb{F}_{p}\right) \otimes H^{*}\left(\Omega S^{n} ; \mathbb{F}_{p}\right) \cong H^{*}\left(F ; \mathbb{F}_{p}\right) .
\end{aligned}
$$

\subsection{The homotopy fiber of the Freudenthal suspension map}

Let $X$ be a path-connected space. Consider the map $e: \Sigma X @>>>\Sigma \Omega \Sigma X$ obtained by suspending the adjunction $X @>>>\Omega \Sigma X$. Recall that there is a homotopy equivalence $h: \Sigma \Omega \Sigma X @>>>\Sigma\left(\bigvee_{k \geqslant 1} X^{(k)}\right)$, where $X^{(k)}$ denotes the $k$ th iterated smash product of $X$ with itself, and that he is homotopic to the inclusion $i: \Sigma X \hookrightarrow \Sigma\left(\bigvee_{k \geqslant 1} X^{(k)}\right)$. The homotopy fiber of $e$ is therefore homotopic to the homotopy fiber of $i$, which we denote $F$.

Recall the result due to Bott and Samelson (see, e.g., $[\mathbf{1 3}, 7.3])$ that $H_{*}(\Omega \Sigma X ; R) \cong$ $T\left(\tilde{H}_{*}(X ; R)\right)$ as algebras, for any PID $R$ and any connected space $X$. Furthermore, the adjuntion $X @>>>\Omega X$ induces the natural inclusion $\tilde{H}_{*}(X ; R) \hookrightarrow$ $T\left(\tilde{H}_{*}(X ; R)\right)$.

In this example we provide a model for the homotopy fiber of $e$ when $X=S^{n}$, $n \geqslant 1$, so that the inclusion we must model is

$$
i: S^{n+1} \hookrightarrow \bigvee_{k \geqslant 1} S^{k n+1} .
$$

Thanks to the result of Bott and Samelson, it is easy to see that this map is $\mathbb{Z}$ formalizable, with strict Hopf model

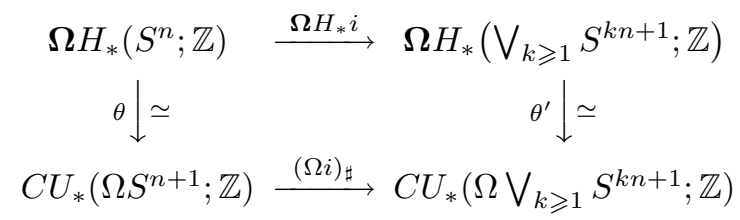

where $\theta^{\prime}$, which is an extension of $\theta$, sends a generator of $H_{*}\left(S^{m n+1} ; \mathbb{Z}\right)$ to a repre- 
sentative of the corresponding algebra generator of the free algebra

$$
H_{*}\left(\Omega \bigvee_{k \geqslant 1} S^{k n+1} ; \mathbb{Z}\right) \cong T\left(\tilde{H}_{*}\left(\bigvee_{k \geqslant 1} S^{k n+1} ; \mathbb{Z}\right)\right)
$$

When we apply Corollary 3.4 to (4.2), we obtain a model

$$
\zeta: \mathcal{M}=\left(\mathbb{Z}\left[v_{1}\right] /\left(v_{1}^{2}\right) \otimes \perp\left(s v_{1}, s v_{2}, s v_{3}, \ldots\right), D\right) @>\simeq>>C^{*}(F ; \mathbb{Z})
$$

where $\left|v_{k}\right|=k n+1$, so that $\left|s v_{k}\right|=k n$. The differential satisfies

$$
D\left(1 \otimes s v_{k_{1}} \cdots s v_{k_{m}}\right)= \begin{cases}-(-1)^{\varepsilon} v_{1} \otimes s v_{k_{1}} \cdots s v_{k_{m-1}} & \text { if } k_{m}=1 \\ 0 & \text { else }\end{cases}
$$

where $\epsilon=n^{2} \cdot \sum_{j=1}^{m-1} k_{j}$, and

$$
D\left(v_{1} \otimes s v_{k_{1}} \cdots s v_{k_{m}}\right)=0
$$

for all $k_{1}, \ldots, k_{m}$ and for all $m \geqslant 0$.

By the argument at the end of the proof of Proposition $3.2, H^{*} \mathcal{M} \cong H^{*}(F ; \mathbb{Z})$ as algebras, since the inclusion $i: S^{n+1} \hookrightarrow \bigvee_{k \geqslant 1} S^{k n+1}$ admits an obvious retraction. Thus, if $V=\bigoplus_{k \geqslant 1} \mathbb{Z} \cdot s v_{k}$ and $\bar{V}=\bigoplus_{k \geqslant 2} \mathbb{Z} \cdot s v_{k}$, then

$$
H^{*}(F ; \mathbb{Z}) \cong(\perp V) \otimes \bar{V}
$$

\section{References}

[1] Adams, J. F. On the cobar construction, Proc. Nat. Acad. Sci. USA, 42(1956), 409-412

[2] Anick, D. Hopf algebras up to homotopy, J. Amer. Math. Soc., textbf2(1989), 417-453

[3] Adams, J. F., Hilton, P. J. On the chain algebra of a loop space, Comm. Math. Helv., 30(1955), 305-330

[4] Dupont, N. R-formalité des algèbres et des espaces, Modèles algébrique en topologie, Thèse d'habilitation, Université de Lille, 1997

[5] Dupont, N., Hess, K. Noncommutative algebraic models for fiber squares, Math. Annalen, 314(1999), 449-467

[6] Dupont, N., Hess, K. How to model the free loop space algebraically, Math. Annalen, 314(1999), 469-490

[7] Dupont, N., Hess, K. Commutative free loop space models at large primes to appear in Math. Zeitschrift

[8] Dupont, N., Hess, K. Hochschild cohomology is topological to appear in J. Pure Appl. Algebra

[9] Félix, Y., Halperin, S., Thomas, J.-C. Adams' cobar equivalence, Trans. Amer. Math. Soc. 329(1992), 531-549

[10] Félix, Y., Halperin, S., Thomas, J.-C. Differential graded algebras in topology, Handbook of Algebraic Topology, Elsevier Science B. V., 1995, 829-866 
[11] Halperin, S. Universal enveloping algebras and loop space homology, J. Pure Applied Algebra, 83(1992), 237-282

[12] McCleary, J. A User's Guide to Spectral Sequences, Second Edition, Cambridge University Press, 2001

[13] Selick, P. Introduction to Homotopy Theory, Fields Institute Monographs, American Mathematical Society, 9(1997)

This article may be accessed via WWW at http://www.rmi.acnet.ge/hha/ or by anonymous ftp at

$$
\text { ftp://ftp.rmi.acnet.ge/pub/hha/volumes/2002/n2a5/v4n2a5.(dvi,ps,pdf) }
$$

Nicolas Dupont Nicolas.Dupont@univ-lille1.fr

Université de Lille

F-59655 Villeneuve d'Ascq

France

Kathryn Hess kathryn.hess@epfl.ch

Département de mathématiques,

Ecole Polytechnique Fédérale de Lausanne,

CH-1015 Lausanne,

Switzerland 